\title{
Evaluation of Techniques for Inconsistency Handling in OWL 2 QL Ontologies
}

\author{
Riccardo Rosati, Marco Ruzzi, Mirko Graziosi, and Giulia Masotti \\ DIAG, Sapienza Università di Roma \\ Via Ariosto 25, I-00185 Roma, Italy
}

\begin{abstract}
In this paper we present the Quonto Inconsistent Data handler (QuID). QuID is a reasoner for OWL 2 QL that is based on the system Quonto and is able to deal with inconsistent ontologies. The central aspect of QuID is that it implements two different, orthogonal strategies for dealing with inconsistency: ABox repairing techniques, based on data manipulation, and consistent query answering techniques, based on query rewriting. Moreover, by exploiting the ability of Quonto to delegate the management of the ABox to a relational database system (DBMS), such techniques are potentially able to handle very large inconsistent ABoxes. For the above reasons, QuID allows for experimentally comparing the above two different strategies for inconsistency handling in the context of OWL 2 QL. We thus report on the experimental evaluation that we have conducted using QuID. Our results clearly point out that inconsistency-tolerance in OWL 2 $\mathrm{QL}$ ontologies is feasible in practical cases. Moreover, our evaluation singles out the different sources of complexity for the data manipulation technique and the query rewriting technique, and allows for identifying the conditions under which one method is more efficient than the other.
\end{abstract}

\section{Introduction}

One of the most important current issues in OWL ontology management is dealing with inconsistency, that is, the presence of contradictory information in the ontology [8]. It is well-known that the classical semantics of OWL and Description Logics (DL) is not inconsistency-tolerant, i.e., it does not allow for using in a meaningful way any piece of information in an inconsistent ontology. On the other hand, the size of ontologies used by real applications is scaling up, and ontologies are increasingly merged and integrated into larger ontologies: the probability of creating inconsistent ontologies is consequently getting higher and higher (see e.g. [4]).

In this paper we focus on ABox inconsistency, i.e., the case of inconsistent ontologies where the TBox (intensional part of the ontology) is consistent, while the ABox (extensional part of the ontology) is inconsistent with the TBox, i.e., a subset of the assertions in the ABox contradicts one or more TBox assertions.

We follow an approach that is formally based on inconsistency-tolerant semantics; such semantics overcome the limitations of the classical DL semantics in inconsistency management. In particular, we consider inconsistency-tolerant semantics for general DLs recently proposed in [5], called IAR semantics, for which reasoning has been studied in the context of the Description Logics of the DL-Lite family, and in particular 
the DL DL-Lite $A$, that underlies the OWL profile OWL 2 QL. The IAR semantics is centered around the notion of ABox repair, which is a very simple and natural one: the ABox repair of a DL ontology is the intersection of all the maximal subsets of the ABox that are consistent with the TBox.

Recently, two different methods for reasoning under the $I A R$ inconsistency-tolerant semantics have been studied: techniques based on the computation of the ABox repair (ABox cleaning) and techniques based on a tranformation of the queries posed to the (possibly inconsistent) ontology (consistent query rewriting). In particular, in [5] it was proved that computing the ABox repair of a DL-Lite $A$ ontology under the IAR semantics is a tractable problem. Then, in [6] a technique for query answering under $I A R$-semantics in $D L$-Lite $A$ is presented: instead of modifying the ABox, this method is based on computing a rewriting $Q^{\prime}$ of the initial query $Q$ and then evaluating the query $Q^{\prime}$ with respect to the original ABox.

We argue that the results of [5]6] are potentially very important from the practical viewpoint, for the following reasons: (i) they are based on formally grounded notions of inconsistency-tolerant semantics; (ii) they identify (to the best of our knowledge) the first inconsistency-tolerant semantics in DLs for which query answering is tractable. So, based on such results, in principle it might be possible to define practical algorithms for handling inconsistency in OWL 2 QL.

This paper starts from the above results, and tries to provide an experimental evaluation and comparison of both the ABox cleaning approach and the consistent query rewriting approach mentioned above. In particular, our main goal was to address the following fundamental questions: (i) is ABox cleaning a feasible technique? (ii) is consistent query rewriting a feasible technique? (iii) under which conditions consistent query rewriting is to prefer to ABox cleaning (and vice versa)?

In this paper, we provide the following contributions:

(1) We present effective techniques for both ABox cleaning and consistent query rewriting in DL-Lite $A$ /OWL 2 QL under $I A R$ semantics. To this aim, we present the QUonto Inconsistent Data handler (QuID), that implements, within the Quonto system 1 techniques for both the computation of the ABox repair of a $\mathrm{DL}-\mathrm{Lite}_{A}$ ontology under the above semantics, as well as techniques for computing the consistent query rewriting of queries. QuID constitutes (to the best of our knowledge) the first implementation of tractable algorithms for handling inconsistent instances in OWL ontologies. Moreover, Quonto delegates the management of the ABox to a relational database system (DBMS). Therefore, for ABox cleaning, all modifications of the ABox are delegated to the DBMS through SQL queries and updates; and for consistent query rewriting, the rewritten query can be directly executed by the DBMS on the original database. This potentially allows for handling inconsistency in very large ABoxes under both techniques.

(2) We present the results of a set of experiments that we have conducted using QuID. These results clearly show that ABox cleaning in $D L$-Lite $A$ is actually scalable: QuID is able to efficiently compute the $I A R$ repair of both complex and large ontologies, whose ABoxes contain up to millions of assertions and have hundreds of thousands of assertions inconsistent with the TBox. On the other hand, the results for the query answering

${ }^{1}$ http: //www.dis. uniroma.it/ quonto 
technique based on consistent query rewriting are in general less encouraging, since the structural complexity of the reformulated queries makes the whole query answering process slower than the approach based on ABox cleaning, although consistent query rewriting does not require pre-processing of the ABox.

(3) Our experimental results allow us to understand the actual impact of the different aspects involved in the computation of the ABox repair and in consistent query rewriting, and the limits and possibilities of the two approaches implemented in QuID.

The rest of the paper is organized as follows. In Section 2 we present a detailed algorithm for computing $I A R$ repairs in $D L-$ Lite $_{A}$. In Section 3, we briefly recall the algorithm presented in [6] for consistent query rewriting under $I A R$ semantics in $D L-L_{\text {Lite }}$. In Section 4 we present the QuID system and report on the experimental evaluation we have conducted with QuID. Finally, in Section 5 we conclude the paper.

\section{ABox Cleaning Technique for OWL 2 QL}

We start by briefly recalling the DL $D L$-Lite $A$ and the $I A R$ semantics.

In this paper we consider DL ontologies specified in $D L$-Lite $_{A}$, a member of the DL-Lite family of tractable Description Logics [2]1], which is at the basis of OWL 2 QL, one of the profiles of OWL 2, the ontology specification language of the World Wide Web Consortium (W3C). DL-Lite $A$ distinguishes concepts from value-domains, which denote sets of (data) values, and roles from attributes, which denote binary relations between objects and values. Concepts, roles, attributes, and value-domains in this DL are formed according to the following syntax:

$$
\begin{array}{l|l|l|l|l}
B \longrightarrow A & \exists Q \mid \delta(U) & E \longrightarrow \rho(U) \\
C \longrightarrow B & \neg B & F \longrightarrow T_{D}\left|T_{1}\right| \cdots \mid T_{n} \\
Q \longrightarrow P & P^{-} & V \longrightarrow U \mid \neg U & & \\
R \longrightarrow Q & \neg Q & &
\end{array}
$$

In such rules, $A, P$, and $U$ respectively denote an atomic concept (i.e., a concept name), an atomic role (i.e., a role name), and an attribute name, $P^{-}$denotes the inverse of an atomic role, whereas $B$ and $Q$ are called basic concept and basic role, respectively. Furthermore, $\delta(U)$ denotes the domain of $U$, i.e., the set of objects that $U$ relates to values; $\rho(U)$ denotes the range of $U$, i.e., the set of values that $U$ relates to objects; $\top_{D}$ is the universal value-domain; $T_{1}, \ldots, T_{n}$ are $n$ pairwise disjoint unbounded valuedomains. A $D L$-Lite $_{A}$ ontology is a pair $\mathcal{K}=\langle\mathcal{T}, \mathcal{A}\rangle$, where $\mathcal{T}$ is the TBox and $\mathcal{A}$ the ABox. The TBox $\mathcal{T}$ is a finite set of assertions of the form

$$
B \sqsubseteq C \quad Q \sqsubseteq R \quad E \sqsubseteq F \quad U \sqsubseteq V \quad \text { (funct } Q) \quad \text { (funct } U \text { ) }
$$

From left to right, the first four assertions respectively denote inclusions between concepts, roles, value-domains, and attributes. In turn, the last two assertions denote functionality on roles and on attributes. In fact, in $D L-$ Lite $_{A}$ TBoxes we further impose that roles and attributes occurring in functionality assertions cannot be specialized (i.e., they cannot occur in the right-hand side of inclusions). In practice, the only difference between $D L-$ Lite $_{A}$ and OWL 2 QL lies in the presence of functionality assertions (which 
are not allowed in OWL 2 QL). Due to space limitations, we refer the reader to [7] for details on the semantics of $D L-$ Lite $_{A}$.

We then briefly recall the $I A R$ semantics for inconsistency-tolerance in DL ontologies (see [5] for more details). Let $\mathcal{K}=\langle\mathcal{T}, \mathcal{A}\rangle$ be a DL ontology. Then, the IAR-repair of $\mathcal{K}$ is defined as the ABox corresponding to the intersection of all the maximal subsets of $\mathcal{A}$ that are consistent with $\mathcal{T}$. A first-order formula $\phi$ is entailed by $\mathcal{K}$ under the $I A R$ semantics if $\phi$ is entailed by $\left\langle\mathcal{T}, \mathcal{A}_{R}\right\rangle$ under the standard DL semantics, where $\mathcal{A}_{R}$ is the $I A R$-repair of $\mathcal{K}$. We are interested in checking (Boolean) unions of conjunctive queries (UCQs) over DL ontologies.

The technique for computing the $I A R$-repair of a $D L$-Lite $A$ ontology $\langle\mathcal{T}, \mathcal{A}\rangle$ is based on the idea of deleting from $\mathcal{A}$ all the membership assertions participating in minimal conflict sets for $\mathcal{T}$. As shown in [5], this task is relatively easy (in particular, tractable) in $D L$-Lite ${ }_{A}$ because the following property holds: for every $D L$-Lite $A$ TBox $\mathcal{T}$, all the minimal conflict sets for $\mathcal{T}$ are either unary conflict sets or binary conflict sets. This property is actually crucial for tractability of reasoning under $I A R$ semantics.

We now present a detailed algorithm for computing the $I A R$-repair of a $D L-L_{\text {ite }} A$ ontology. This algorithm exploits the techniques presented in [5], whose aim was only to provide PTIME upper bounds for the problem of computing such repairs. In particular, the present algorithms specify efficient ways of detecting minimal conflict sets. Instead, the previous techniques check all unary and binary subsets of the ABox for these purposes.

In the following, we call annotated ABox assertion an expression $\xi$ of the form $\langle\alpha, \gamma\rangle$ where $\alpha$ is an ABox assertion and $\gamma$ is a value in the set $\{$ cons, ucs, bcs $\}$. Furthermore, we call annotated ABox a set of annotated ABox assertions. The intuition behind an annotated ABox assertion $\xi$ is that its annotation $\gamma$ expresses whether the associated ABox expression $\alpha$ does not participate in any minimal conflict set (cons) or participates in a unary conflict set (ucs) or to a binary conflict set (bcs).

The following algorithm QuID-IAR-repair computes the $I A R$-repair of a DL-Lite $A$ ontology. For ease of exposition, the algorithm does not report details on the treatment of attributes, which are actually handled in a way analogous to roles. In the following, we denote concept names with the symbol $A$, role names with the symbol $P$, basic concepts (that is, a concept name $A$ or the domain of a role $\exists P$ or the range of a role $\exists P^{-}$) with the symbols $B_{1}, B_{2}$, and basic roles (that is, either a role name $P$ or the inverse of a role name $P^{-}$) with the symbols $R, S$. Moreover, the expression $B(a)$ with $B$ basic concept denotes: the instance assertion $A(a)$ if $B=A$; an instance assertion of the form $P(a, b)$ if $B=\exists P$; an instance assertion of the form $P(b, a)$ if $B=\exists P^{-}$.

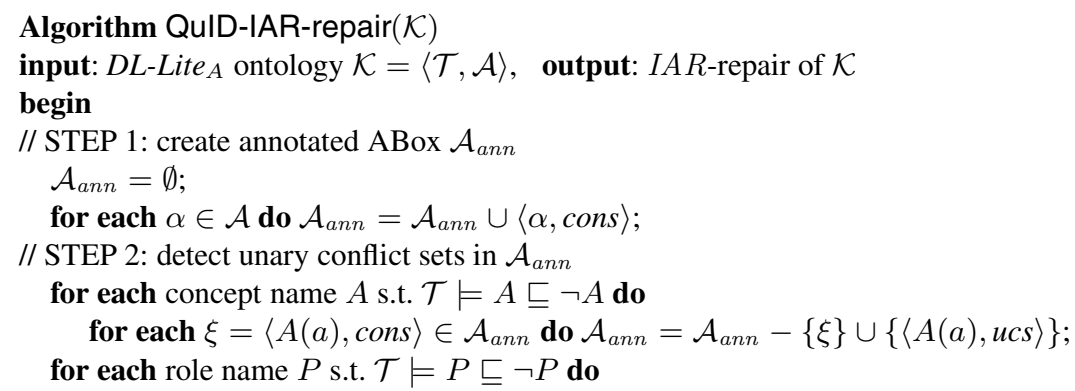




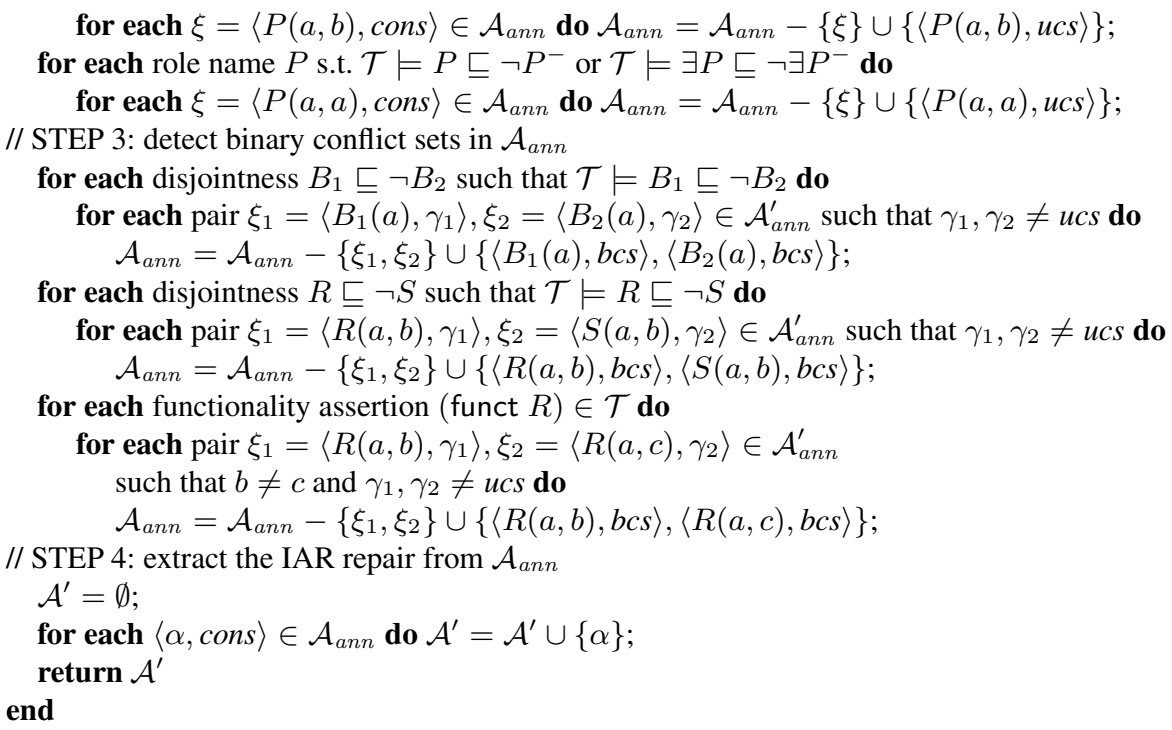

The algorithm QuID-IAR-repair consists of four steps which can be informally described as follows.

step 1. copy of $\mathcal{A}$ into an annotated $A B$ ox $\mathcal{A}_{\text {ann }}$. In this step, the value of the annotation is initialized to cons for all ABox assertions.

step 2. detection of the unary conflict sets in $\mathcal{A}_{\text {ann }}$. For every assertion of the form $\xi=\langle\alpha$, cons $\rangle$, such that $\{\alpha\}$ is a unary conflict set for $\mathcal{T}, \mathcal{A}_{\text {ann }}=\mathcal{A}_{\text {ann }}-\{\xi\} \cup$ $\{\langle\alpha, u c s\rangle\}$, i.e., the annotation relative to $\alpha$ is changed to ucs. Unary conflict sets are actually detected through TBox reasoning, by looking at empty concepts and roles in $\mathcal{T}$, as well as asymmetric roles, i.e., roles disjoint with their inverse.

step 3. detection of the binary conflict sets in $\mathcal{A}_{\text {ann }}$. For every pair of assertions of the form $\xi_{1}=\left\langle\alpha_{1}, \gamma_{1}\right\rangle, \xi_{2}=\left\langle\alpha_{2}, \gamma_{2}\right\rangle$ such that $\gamma_{1} \neq u c s$ and $\gamma_{2} \neq u c s$ and $\{\alpha, \beta\}$ is a binary conflict set for $\mathcal{T}, \mathcal{A}_{\text {ann }}=\mathcal{A}_{\text {ann }}-\left\{\xi_{1}, \xi_{2}\right\} \cup\{\langle\alpha, b c s\rangle,\langle\beta, b c s\rangle\}$, i.e., the annotation relative to $\alpha$ and $\beta$ is changed to bcs. As in the case of unary conflict sets, to find binary conflict sets the algorithm looks for disjoint concepts and roles in $\mathcal{T}$, as well as functional roles.

step 4. extraction of the IAR-repair from $\mathcal{A}_{\text {ann }}$. The $I A R$-repair can be now simply extracted from the annotated ABox $\mathcal{A}_{\text {ann }}$, by eliminating both unary conflict sets and binary conflict sets. Therefore, for every assertion of the form $\langle\alpha$, cons $\rangle$ in $\mathcal{A}_{\text {ann }}, \alpha$ is copied into the (non-annotated) ABox $\mathcal{A}^{\prime}$ which is finally returned by the algorithm.

Correctness of the above algorithm can be proved starting from the results in [5].

Theorem 1. Let $\mathcal{K}$ be a DL-Lite $A$ ontology and let $\mathcal{A}^{\prime}$ be the ABox returned by QuID-IAR-repair $(\mathcal{K})$. Then, $\mathcal{A}^{\prime}$ is the IAR repair of $\mathcal{K}$. 


\section{Perfect Reformulation of UCQs under IAR Semantics}

We now briefly recall the query rewriting technique proposed in [6]. Such a technique computes a first-order query $Q^{\prime}$ starting from a union of conjunctive queries $Q$ and a $D L$-Lite $_{A}$ TBox $\mathcal{T}$. The query $Q^{\prime}$ is a perfect reformulation of $Q$ with respect to $\mathcal{T}$ under the IAR semantics, i.e., $Q^{\prime}$ is such that, for every $\mathrm{ABox} \mathcal{A}$, the answers to $Q$ over $\langle\mathcal{T}, \mathcal{A}\rangle$ under the $I A R$ semantics correspond to to the answers to $Q^{\prime}$ computed over the ABox $\mathcal{A}$ only. Due to space limits, here we just report the main definitions of the query rewriting technique: we refer the reader to [6] for more details on the method.

The first definition that we give can be used to establish whether a certain atom is consistent with the TBox axioms. Let $A$ be an atomic concept in $\Gamma_{\mathcal{O}}$ and $t$ a term (i.e., either a constant or a variable symbol), we pose $\operatorname{Cons}_{A} t_{A}^{\mathcal{T}}(t)=$ false if $\mathcal{T} \models A \sqsubseteq \neg A$, true otherwise. That is, $\operatorname{Cons}_{A} t_{A}^{\mathcal{T}}(t)$ is false if and only if the concept $A$ is unsatisfiable. For an atomic role $P \in \Gamma_{\mathcal{O}}$ and terms $t, t^{\prime}$, we define: (i) $\operatorname{Cons} t_{P}^{\mathcal{T}}\left(t, t^{\prime}\right)=$ false if $\mathcal{T} \models P \sqsubseteq \neg P$; (ii) $t \neq t^{\prime}$ if $\mathcal{T} \models P \sqsubseteq \neg P^{-}$or $\mathcal{T} \models \exists P \sqsubseteq \neg \exists P^{-}$; (iii) true otherwise (an analogous definition holds for an attribute $U \in \Gamma_{\mathcal{O}}$ and terms $t$ and $t^{\prime}$ ).

Now we deal with possible clashes involving negative inclusions, which are also called disjointnesses. Let $B$ be a basic concept built from an atomic concept or an atomic role of $\Gamma_{\mathcal{O}}$, and let $t$ be a term. Then, we define $\operatorname{NotDisjClash}_{B}^{\mathcal{T}}(t)$ as the following FOL formula:

$$
\begin{aligned}
& \bigwedge_{A \in D C N(B, \mathcal{T})} \neg\left(A(t) \wedge \operatorname{Cons}_{A} t_{A}^{\mathcal{T}}(t)\right) \wedge \bigwedge_{P \in D R D(B, \mathcal{T})} \neg\left(\exists y \cdot P(t, y) \wedge \operatorname{Cons} t_{P}^{\mathcal{T}}(t, y)\right) \wedge \\
& \bigwedge_{P \in D R R(B, \mathcal{T})} \neg\left(\exists y \cdot P(y, t) \wedge \operatorname{Cons} t_{P}^{\mathcal{T}}(y, t)\right) \wedge \bigwedge_{U \in D A D(B, \mathcal{T})} \neg\left(\exists y \cdot U(t, y) \wedge \operatorname{ConsAt}_{U}^{\mathcal{T}}(t, y)\right)
\end{aligned}
$$

where $y$ is a variable symbol such that $y \neq t, D C N, D R D, D R R$, and $D A D$ are defined as follows:

$$
\begin{aligned}
& \operatorname{DCN}(B, \mathcal{T})=\left\{A \mid A \text { is an atomic concept of } \Gamma_{\mathcal{O}} \text { and } \mathcal{T} \models B \sqsubseteq \neg A\right\} \\
& D R D(B, \mathcal{T})=\left\{P \mid P \text { is an atomic role of } \Gamma_{\mathcal{O}} \text { and } \mathcal{T} \models B \sqsubseteq \neg \exists P\right\} \\
& \operatorname{DRR}(B, \mathcal{T})=\left\{P \mid P \text { is an atomic role of } \Gamma_{\mathcal{O}} \text { and } \mathcal{T} \models B \sqsubseteq \neg \exists P^{-}\right\} \\
& D A D(B, \mathcal{T})=\left\{U \mid U \text { is an attribute of } \Gamma_{\mathcal{O}} \text { and } \mathcal{T} \models B \sqsubseteq \neg \delta(U)\right\}
\end{aligned}
$$

Let us now consider disjointness clashes for roles. Let $P$ be a role name from $\Gamma_{\mathcal{O}}$ and let $t, t^{\prime}$ be terms, we define the formula $\operatorname{NotDisjClash}_{P}^{\mathcal{T}}\left(t, t^{\prime}\right)$ as follows:

$$
\begin{aligned}
& \bigwedge_{S \in \operatorname{DisjRoles}(P, \mathcal{T})} \neg\left(S\left(t, t^{\prime}\right) \wedge \operatorname{ConsA}_{S}^{\mathcal{T}}\left(t, t^{\prime}\right)\right) \wedge \operatorname{NotDisjClash}_{\exists P}^{\mathcal{T}}(t) \wedge \\
& \bigwedge_{S \in \operatorname{DisjInvRoles}(P, \mathcal{T})} \neg\left(S\left(t^{\prime}, t\right) \wedge \operatorname{ConsAt}_{S}^{\mathcal{T}}\left(t^{\prime}, t\right)\right) \wedge \operatorname{NotDisjClash}_{\exists P^{-}}^{\mathcal{T}}\left(t^{\prime}\right)
\end{aligned}
$$

where, again, if either $t$ or $t^{\prime}$ are variable symbols, then they are free variables, and the sets $\operatorname{DisjRoles}(P, \mathcal{T})$ and DisjInvRoles $(P, \mathcal{T})$ are defined as follows:

$\operatorname{DisjRoles}(P, \mathcal{T})=\left\{S \mid S\right.$ is a role name of $\Gamma_{\mathcal{O}}$ and $\left.\mathcal{T} \models P \sqsubseteq \neg S\right\}$

$\operatorname{DisjInvRoles}(P, \mathcal{T})=\left\{S \mid S\right.$ is a role name of $\Gamma_{\mathcal{O}}$ and $\left.\mathcal{T} \models P \sqsubseteq \neg S^{-}\right\}$.

Intuitively, $\operatorname{NotDisjClash}_{P}^{\mathcal{T}}\left(t, t^{\prime}\right)$ will be used in the reformulation to deal with possible violations of negative inclusions involving $P$. This means considering role inclusions, through the sets $\operatorname{DisjRoles}(P, \mathcal{T})$ and $\operatorname{DisjInvRoles}(P, \mathcal{T})$, and concept inclusions of the form $\exists P \sqsubseteq \neg B$ and of the form $\exists P^{-} \sqsubseteq \neg B$, through the use 
of NotDisjClash ${ }_{\exists P}^{\mathcal{T}}(t)$ and NotDisjClash $\mathcal{T}_{\exists P^{-}}^{\mathcal{T}}\left(t^{\prime}\right)$, respectively. $\operatorname{Cons} t_{S}^{\mathcal{T}}\left(t, t^{\prime}\right)$ plays here a role analogous to the one played by ConsAt formulas in $\operatorname{NotDisjClash}_{B}^{\mathcal{T}}(t)$. (The function NotDisjClash $\mathcal{T}_{U}^{\mathcal{T}}$ for attributes $U$ is defined in an analogous way.)

Finally, we consider clashes on functionalities and define $\operatorname{NotFunctClash}_{P}^{\mathcal{T}}\left(t, t^{\prime}\right)$ as the following FOL formula:

- if (funct $P) \notin \mathcal{T}$ and (funct $\left.P^{-}\right) \notin \mathcal{T}$, then NotFunctClash ${ }_{P}^{\mathcal{T}}\left(t, t^{\prime}\right)=$ true $_{\mathcal{T}}$;

- if (funct $P$ ) $\in \mathcal{T}$ and (funct $P^{-}$) $\notin \mathcal{T}$, then $\operatorname{NotFunctClash}_{P}^{\mathcal{T}}\left(t, t^{\prime}\right)=$ $\neg\left(\exists y . P(t, y) \wedge y \neq t^{\prime} \wedge \operatorname{Cons} t_{P}^{\mathcal{T}}(t, y)\right) ;$

- if (funct $P$ ) $\notin \mathcal{T}$ and (funct $P^{-}$) $\in \mathcal{T}$, then $\operatorname{NotFunctClash}_{P}^{\mathcal{T}}\left(t, t^{\prime}\right)=$ $\neg\left(\exists y . P\left(y, t^{\prime}\right) \wedge y \neq t \wedge \operatorname{ConsAt}_{P}^{\mathcal{T}}(y, t)\right) ;$

- if (funct $P$ ) $\in \mathcal{T}$ and (funct $P^{-}$) $\in \mathcal{T}$, then NotFunctClash $\operatorname{Not}_{P}^{\mathcal{T}}\left(t, t^{\prime}\right)=$ $\neg\left(\exists y . P(t, y) \wedge y \neq t^{\prime} \wedge \operatorname{Cons} A t_{P}^{\mathcal{T}}(t, y)\right) \wedge \neg\left(\exists y . P\left(y, t^{\prime}\right) \wedge y \neq t \wedge \operatorname{ConsAt}_{P}^{\mathcal{T}}(y, t)\right)$.

(The function NotFunctClash $\mathcal{T}_{U}^{\mathcal{T}}$ for attributes $U$ is defined analogously.)

We are now able to define for each $D L-$ Lite $_{A}$ construct the formula that combines together the various formulas we have introduced for dealing with the various possible clashes: (i) $\operatorname{NotClash}_{A}^{\mathcal{T}}(t)=\operatorname{NotDisjClash}_{A}^{\mathcal{T}}(t)$ for an atomic concept name $A$ and term $t$; (ii) $\operatorname{NotClash}_{Z}^{\mathcal{T}}\left(t, t^{\prime}\right)=N_{\text {otDisjClash }}^{\mathcal{T}}\left(t, t^{\prime}\right) \wedge \operatorname{NotFunctClash}_{Z}^{\mathcal{T}}\left(t, t^{\prime}\right)$ for a role or attribute name $Z$ and terms $t, t^{\prime}$.

Let $q$ be a CQ $\exists x_{1}, \ldots, x_{k} \cdot \bigwedge_{i=1}^{n} A_{i}\left(t_{i}^{1}\right) \wedge \bigwedge_{i=1}^{m} P_{i}\left(t_{i}^{2}, t_{i}^{3}\right) \wedge \bigwedge_{i=1}^{\ell} U_{i}\left(t_{i}^{4}, t_{i}^{5}\right)$, where every $A_{i}$ is an atomic concept, every $P_{i}$ is an atomic role, every $U_{i}$ is an attribute, and every $t_{i}^{1}, t_{i}^{2}, t_{i}^{3}, t_{i}^{4}, t_{i}^{5}$ is either a constant or a variable $x_{j}$ with $1 \leq j \leq k$. Then, we define IncRewriting IAR $(q, \mathcal{T})$ as the following FOL sentence

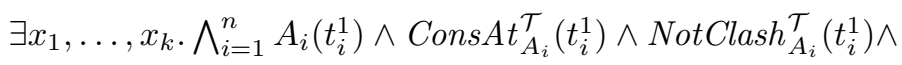

$$
\begin{aligned}
& \bigwedge_{i=1}^{m} P_{i}\left(t_{i}^{2}, t_{i}^{3}\right) \wedge \operatorname{ConsAt}_{P_{i}}^{\mathcal{T}}\left(t_{i}^{2}, t_{i}^{3}\right) \wedge \operatorname{NotClash}_{P_{i}}^{\mathcal{T}}\left(t_{i}^{2}, t_{i}^{3}\right) \\
& \bigwedge_{i=1}^{\ell} U_{i}\left(t_{i}^{4}, t_{i}^{5}\right) \wedge \operatorname{ConsA}_{U_{i}}^{\mathcal{T}}\left(t_{i}^{4}, t_{i}^{5}\right) \wedge \operatorname{NotClash}_{U_{i}}^{\mathcal{T}}\left(t_{i}^{4}, t_{i}^{5}\right)
\end{aligned}
$$

Informally, for each atom $A_{i}\left(t_{i}^{1}\right)$, each membership assertion of the $\mathrm{ABox} \mathcal{A}$ constituting an image of $A_{i}\left(t_{i}^{1}\right)$ has not to be inconsistent with the TBox (condition Cons $A t_{A_{i}}^{\mathcal{T}}\left(t_{i}^{1}\right)$ ), and has not to be involved in any clash with some other assertion of $\mathcal{A}$ on any negative inclusion (condition $\operatorname{NotClash}_{A_{i}}^{\mathcal{T}}\left(t_{i}^{1}\right)$ ). Similarly for atoms of the form $P_{i}\left(t_{i}^{2}, t_{i}^{3}\right)$ and $U_{i}\left(t_{i}^{4}, t_{i}^{5}\right)$.

Let $Q$ be the UCQ $q_{1} \vee \ldots \vee q_{n}$. Then, we define IncRewriting $U C Q_{\text {IAR }}(Q, \mathcal{T})=$ $\bigvee_{i=1}^{n} \operatorname{IncRewriting} \operatorname{IAR}_{(}\left(q_{i}, \mathcal{T}\right)$. Finally, we define $\operatorname{PerfectRef}_{I A R}(Q, \mathcal{T})$ as IncRewritingUC $Q_{I A R}(\operatorname{PerfectRef}(Q, \mathcal{T}), \mathcal{T})$, where $\operatorname{PerfectRef}(Q, \mathcal{T})$ denotes the algorithm for computing a perfect reformulation of a UCQ $Q$ with respect to a $D L-$ Lite $_{A}$ TBox $\mathcal{T}$ under standard semantics [2] (the algorithm $\operatorname{PerfectRef}(Q, \mathcal{T})$ returns a UCQ specified over $\mathcal{T}$ ). It can be shown (see [6]) that $\operatorname{PerfectRef}_{\text {IAR }}(Q, \mathcal{T})$ constitutes a perfect reformulation of $Q$ with respect to $\mathcal{T}$ under $I A R$ semantics.

Therefore, using this technique, it is possible to solve query answering under $I A R$ semantics in $D L$-Lite $_{A}$ as follows. Given the initial query $Q$ and the ontology $\langle\mathcal{T}, \mathcal{A}\rangle$, the first-order query PerfectRef $f_{I A R}(Q, \mathcal{T})$ is computed, and then such a first-order query is evaluated over the original ABox (which is in general inconsistent with $\mathcal{T}$ ). So, in this case no repair of the ABox is performed, differently from the algorithm presented in the previous section. 


\section{Experiments}

We have implemented the techniques presented in the previous Section in the Quonto system, in a module called QuID (the QUonto Inconsistent Data handler). Essentially, QuID is a Java implementation of the above algorithms for ABox repair and for query rewriting. In fact, in the Quonto architecture, the management of the ABox is delegated to a relational database management system (DBMS): therefore, all the operations on ABox assertions of the algorithms for computing repairs are executed in QuID by the DBMS used by Quonto, through appropriate SQL scripts.

We have experimented QuID in order to answer several open questions about: (i) the computational cost of the various steps of the ABox cleaning algorithm and of the query rewriting algorithm; (ii) the scalability of such algorithms; (iii) the impact of the "degree of inconsistency" of the ABox on the computational cost of the algorithms; (iv) the practical difference between the ABox cleaning tecnhique and the purely intensional rewriting technique.

Experimenting the QuID-IAR-repair algorithm. We have experimented our implementation of the QuID-IAR-repair algorithm over the LUBM benchmark ontology whose TBox has 43 concept names, 25 role names, 7 attribute names, and about 200 TBox assertions. We have generated 4 different ABoxes by means of the UBA Data Generator provided by the LUBM website, with an increasing number of assertions, and used such ABoxes in our experiments. It is important to note that the original LUBM ontology has no axioms which can generate inconsistency, and hence, no inconsistent data is contained in the generated ABoxes. So, we sligthly modified the LUBM ontology by adding some "inconsistency-generating" axioms and then added inconsistencies to the ABoxes. We created four different version for every original ABox with different percentages of ABox assertions involved in minimal conflict sets, in order to get ABoxes with respectively $1 \%, 5 \%, 10 \%$ and $20 \%$ of inconsistent assertions, uniformly distributed among the axioms which might generate inconsistency. Figure 1 shows the size (number of instance assertions) of the ABoxes we used in the experiments: every column is labeled with the number of Universities the ABox data contains, and every row is labeled with the percentage of inconsistent facts added to the ABox itself.

Figure 2 report some of the experimental results that we have obtained. The table displayed presents the experimental results for QuID-IAR-repair using a PostgreSQL 9.1 instance as external DBMS. The results have been conducted on a Pentium i5 (2.4 $\mathrm{GHz}$ ) CPU with 4GB RAM under Windows 7 (64 bit) operating system.

All the necessary software, as well as instructions on how to reproduce the experiments presented in this section, are publicly available at http://www.dis.uniroma1.it/ ruzzi/quid/. Further details on the ontology used in the experiments are also available there.

In the table displayed in Figure 2, the first column reports the number of universities represented in the ABox, while the second column reports the percentage of ABox assertions that participate in minimal conflict sets for the considered TBox. Moreover:

- T1 denotes the time to create the annotated ABox (step 1 of QuID-IAR-repair);

2 http://swat.cse. lehigh. edu/projects/lubm/ 
- T2 denotes the time to detect unary and binary conflict sets (steps 2 and 3 of QuIDIAR-repair);

- T3 denotes the time to extract the $I A R$-repair from the annotated ABox (step 4 of QuID-IAR-repair);

- Total is the total time to compute the $I A R$-repair, i.e., T1+T2+T3.

\begin{tabular}{|c|c|c|c|c|c|}
\hline & \multicolumn{4}{|c|}{ Number of Universities } \\
\hline & & 1 & 5 & 10 & 20 \\
\hline \multirow{4}{*}{ 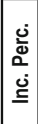 } & 1 & 103765 & 631960 & 1285244 & 2711216 \\
\hline & 5 & 109165 & 658980 & 1339304 & 2819337 \\
\hline & 10 & 115845 & 692400 & 1406124 & 2952957 \\
\hline & 20 & 130445 & 765380 & 1552104 & 3244937 \\
\hline
\end{tabular}

Fig. 1. Size of the UBA generated ABoxes

\begin{tabular}{|r|r|r|r|r|r|}
\hline \#Univ & Inc\% & \multicolumn{1}{|c|}{$\mathrm{T} 1$ (ms) } & T2 (ms) & \multicolumn{1}{c|}{ T3 (ms) } & Total (ms) \\
\hline \multirow{4}{*}{1} & 1 & 66908 & 2356 & 73617 & 142881 \\
\cline { 2 - 6 } & 5 & 69748 & 11559 & 71401 & 152708 \\
\cline { 2 - 6 } & 10 & 71402 & 24523 & 70231 & 166156 \\
\cline { 2 - 6 } & 20 & 85878 & 50014 & 68156 & 204048 \\
\hline \multirow{4}{*}{5} & 1 & 414477 & 13416 & 418970 & 846863 \\
\cline { 2 - 6 } & 5 & 419298 & 60434 & 414854 & 894586 \\
\cline { 2 - 6 } & 10 & 412371 & 131805 & 403619 & 947795 \\
\cline { 2 - 6 } & 20 & 466363 & 254000 & 406880 & 1127243 \\
\hline \multirow{4}{*}{10} & 1 & 968123 & 31060 & 953037 & 1952220 \\
\cline { 2 - 6 } & 5 & 945471 & 140447 & 917890 & 2003808 \\
\cline { 2 - 6 } & 10 & 936688 & 271830 & 884835 & 2093353 \\
\hline \multirow{4}{*}{20} & 20 & 987216 & 573020 & 873664 & 2433900 \\
\cline { 2 - 6 } & 1 & 2381829 & 137327 & 2379121 & 4898277 \\
\cline { 2 - 6 } & 5 & 2485267 & 353486 & 2251335 & 5090088 \\
\cline { 2 - 6 } & 10 & 2233066 & 722468 & 2212381 & 5167915 \\
\hline
\end{tabular}

Fig. 2. Repair generation time

The above experimental results show that:

(i) the computation of the $I A R$-repair (column T1) seems really scalable, and grows almost linearly w.r.t. the size of the ABox.

(ii) the percentage of inconsistency, i.e., the fraction of ABox assertions that participate in minimal conflict sets, has a real impact only on the detection of minimal conflict sets (column T2);

(iii) most of the whole execution time of the QuID-IAR-repair algorithm is devoted to the creation of annotated ABox (T1) and of the final repair (T3): if this could be avoided (e.g., by just modifying the original database, as explained below), the algorithm would be much more efficient, since only time T2 would be consumed.

Experimenting the Consistent Query Rewriting Approach. As above observed, most of the execution time of the algorithm QuID-IAR-repair using a disk-resident DB is due to the creation of the annotated ABox (step 1) and to the creation of the IAR-repair (step 4). Thus, avoiding these steps would dramatically improve the efficiency of this algorithm. 
To this aim, we observe that both the above steps could be completely avoided if the database schema used for representing the ABox would present an additional attribute for storing annotations in every relation (the usual DB representation of an ABox uses a unary relation for every concept and a binary relation for every role). This corresponds to the idea of directly using an annotated ABox instead of a standard ABox in the system. In this case, the computation of the $I A R$-repair could only consist of steps 2 and 3 of the algorithm QuID-IAR-repair. However, the choice of using an annotated ABox instead of a standard ABox could affect query answering, since the queries evaluated on an annotated ABox should be able to only consider the assertions whose annotation is equal to cons. Similarly, exploiting the query rewriting technique presented in Section 3 , it is possible to completely avoid the computation of the annotated ABox, and could be able to evaluate the first-order query corresponding to the perfect reformulation of the original query directly over the original, inconsistent, ABox.

We have experimented whether this choice is actually feasible. In particular, we tested and compared three different approaches: $(I A R)$ evaluation of the $I A R$ perfect reformulation over the inconsistent ABox; $(A N N)$ evaluation over the annotated ABox $\mathcal{A}_{\text {ann }}$ (produced by the QuID-IAR-repair algorithm) of the original query enriched with suitable conditions that are needed to filter out the assertions belonging to minimal conflict sets; $(R E P)$ evaluation of the original query over the repair using the standard query answering technique of QuOnto. Figure 3 presents a table showing the evaluation time of nine of the fourteen queries of the LUBM benchmark over all the ABoxes previously considered. We adopted a timeout (denoted by T.o. in the table) of 1 hour

Comparing the Two Approaches. These experimental results show that, in QuID, evaluating queries on the annotated ABox is computationally not harder than evaluating them on the standard ABox. Conversely, the evaluation of the $I A R$ perfect reformulations is often more expensive (in particular, it is more expensive for queries Q5-Q9). This is due to the fact that we have built no repair and we are querying the inconsisent ABox: thus, as shown in the previous section, the $I A R$ perfect reformulation essentially has to select only assertions of the ABox which do not participate in minimal inconsistent sets (with respect to the TBox). This makes the form of such queries quite involved: in particular, the SQL queries corresponding to the $I A R$ perfect refomulations of UCQs may present several nesting levels, which makes such queries hard to evaluate by current DBMSs. This consideration is enforced, e.g., by the evaluation time of query Q5, which is greater than 1 hour on the ABox representing 5 universities. That is, in this case the time to evaluate the $I A R$ perfect reformulation of this query over the original ABox is much greater than computing the $I A R$ repair and then evaluating the original query on the repaired ABox.

Combining the results of Figure 2 and Figure 3 it seems that, in general, the ABox cleaning approach is more convenient than the consistent query rewriting approach. In other words, the cost of preprocessing the ABox is generally an acceptable one, and really pays off during the evaluation of the queries, especially when the annotated representation of the ABox is adopted.

\footnotetext{
${ }^{3}$ Further details on our experiments can be found at http://www.dis.uniroma1.it/ ruzzi/quid/
} 


\begin{tabular}{|c|c|c|c|c|c|c|c|c|c|c|}
\hline & & & Q1 & & & Q2 & & & Q3 & \\
\hline \#Univ & $\operatorname{lnc} \%$ & $I A R$ & ANN & $R E P$ & $I A R$ & ANN & REP & $I A R$ & ANN & REP \\
\hline \multirow{4}{*}{1} & 1 & 2324 & 37 & 31 & 31 & 2 & 0 & 32 & 7 & 16 \\
\hline & 5 & 2340 & 36 & 16 & 32 & 3 & 0 & 31 & 8 & 0 \\
\hline & 10 & 2325 & 40 & 16 & 31 & 0 & 0 & 31 & 10 & 0 \\
\hline & 20 & 2340 & 36 & 16 & 32 & 3 & 0 & 31 & 7 & 0 \\
\hline \multirow{4}{*}{5} & 1 & 905 & 2393 & 2808 & 31 & 238 & 405 & 63 & 874 & 999 \\
\hline & 5 & 874 & 2882 & 3135 & 16 & 162 & 297 & 62 & 964 & 936 \\
\hline & 10 & 561 & 3826 & 2668 & 32 & 113 & 218 & 78 & 882 & 936 \\
\hline & 20 & 942 & 2968 & 4259 & 31 & 423 & 390 & 63 & 1063 & 1435 \\
\hline \multirow{4}{*}{10} & 1 & 6661 & 11659 & 9531 & 32 & 162 & 390 & 3510 & 2441 & 2434 \\
\hline & 5 & 4306 & 10878 & 9610 & 32 & 355 & 281 & 2122 & 2111 & 1966 \\
\hline & 10 & 5663 & 8928 & 6926 & 47 & 437 & 406 & 1856 & 1977 & 2074 \\
\hline & 20 & 4540 & 7677 & 7425 & 62 & 367 & 281 & 2871 & 1694 & 1701 \\
\hline \multirow{4}{*}{20} & 1 & 11170 & 13625 & 20748 & 32 & 210 & 375 & 1639 & 1060 & 3229 \\
\hline & 5 & 9859 & 18356 & 21887 & 63 & 317 & 390 & 2028 & 2198 & 3323 \\
\hline & 10 & 9844 & 16870 & 14883 & 47 & 365 & 249 & 2075 & 2605 & 2996 \\
\hline & 20 & 8783 & 18347 & 15725 & 63 & 482 & 296 & 2496 & 1486 & 2402 \\
\hline & & \multicolumn{3}{|c|}{ Q4 } & \multicolumn{3}{|c|}{ Q5 } & \multicolumn{3}{|c|}{ Q6 } \\
\hline \#Univ & $\operatorname{lnc} \%$ & $I A R$ & ANN & $R E P$ & IAR & ANN & $R E P$ & $I A R$ & ANN & $R E P$ \\
\hline \multirow{4}{*}{1} & 1 & 78 & 4 & 0 & 4898 & 17 & 15 & 297 & 7 & 16 \\
\hline & 5 & 78 & 0 & 15 & 4899 & 20 & 16 & 312 & 10 & 0 \\
\hline & 10 & 78 & 0 & 0 & 4696 & 10 & 15 & 312 & 0 & 0 \\
\hline & 20 & 62 & 4 & 15 & 4727 & 18 & 16 & 312 & 7 & 0 \\
\hline \multirow{4}{*}{5} & 1 & 125 & 446 & 453 & T.o. & 15998 & 17659 & 1529 & 35 & 47 \\
\hline & 5 & 203 & 351 & 421 & T.o. & 23721 & 14914 & 1529 & 37 & 47 \\
\hline & 10 & 187 & 348 & 561 & T.o. & 15243 & 16521 & 1560 & 31 & 31 \\
\hline & 20 & 202 & 444 & 749 & T.o. & 19612 & 22963 & 1748 & 30 & 15 \\
\hline \multirow{4}{*}{10} & 1 & 3588 & 220 & 1372 & T.o. & 54142 & 52434 & 2995 & 66 & 62 \\
\hline & 5 & 889 & 912 & 1185 & T.o. & 41709 & 54600 & 3167 & 66 & 62 \\
\hline & 10 & 1701 & 237 & 936 & T.o. & 50099 & 48875 & 3229 & 70 & 63 \\
\hline & 20 & 1607 & 771 & 843 & T.o. & 35762 & 40138 & 3448 & 73 & 78 \\
\hline \multirow{4}{*}{20} & 1 & 1965 & 1398 & 1794 & T.o. & 99222 & 127796 & 6396 & 157 & 156 \\
\hline & 5 & 2106 & 1121 & 1435 & T.o. & 112814 & 132288 & 6536 & 152 & 141 \\
\hline & 10 & 2262 & 1238 & 1357 & T.o. & 113969 & 110622 & 6739 & 157 & 156 \\
\hline & 20 & 3900 & 1273 & 1544 & T.o. & 103710 & 110339 & 7332 & 145 & 140 \\
\hline & & \multicolumn{3}{|c|}{ Q7 } & \multicolumn{3}{|c|}{ Q8 } & \multicolumn{3}{|c|}{ Q9 } \\
\hline \#Univ & $\operatorname{lnc} \%$ & $I A R$ & $A N N$ & $R E P$ & $I A R$ & ANN & $R E P$ & $I A R$ & ANN & $R E P$ \\
\hline \multirow{4}{*}{1} & 1 & 4539 & 37 & 31 & 499 & 8 & 16 & 219 & 3 & 0 \\
\hline & 5 & 4695 & 40 & 47 & 515 & 0 & 0 & 187 & 0 & 15 \\
\hline & 10 & 4586 & 40 & 31 & 500 & 10 & 16 & 171 & 0 & 0 \\
\hline & 20 & 4571 & 38 & 47 & 406 & 9 & 0 & 140 & 2 & 0 \\
\hline \multirow{4}{*}{5} & 1 & 4652 & 557 & 453 & 1716 & 43 & 31 & 172 & 3 & 31 \\
\hline & 5 & 4664 & 575 & 343 & 1731 & 39 & 31 & 156 & 29 & 0 \\
\hline & 10 & 4648 & 533 & 515 & 1732 & 57 & 31 & 156 & 9 & 47 \\
\hline & 20 & 4665 & 828 & 671 & 1731 & 40 & 16 & 141 & 30 & 31 \\
\hline \multirow{4}{*}{10} & 1 & 4901 & 799 & 593 & 4025 & 71 & 62 & 234 & 17 & 16 \\
\hline & 5 & 4790 & 894 & 655 & 3479 & 80 & 63 & 219 & 20 & 31 \\
\hline & 10 & 4695 & 813 & 686 & 3339 & 88 & 63 & 234 & 18 & 15 \\
\hline & 20 & 4477 & 833 & 483 & 3354 & 85 & 63 & 156 & 13 & 15 \\
\hline & 1 & 4508 & 590 & 577 & 5444 & 160 & 141 & 296 & 15 & 15 \\
\hline 20 & 5 & 4509 & 898 & 733 & 5553 & 160 & 140 & 312 & 10 & 16 \\
\hline & 10 & 4430 & 753 & 437 & 5974 & 162 & 141 & 297 & 21 & 15 \\
\hline & 20 & 4508 & 839 & 437 & 12215 & 171 & 156 & 156 & 9 & 16 \\
\hline
\end{tabular}

Fig. 3. Query answering time (in milliseconds) for the various techniques 
On the other hand, it is worth recalling that the ABox cleaning approach might not always be possible or easily realizable in real applications, especially in ontology-based data access (OBDA) scenarios where the ABox is actually a virtual object that is defined through virtual queries/views over one or more remote databases: (see e.g., [7]): in these cases, the OBDA system can typically only read such databases.

\section{Conclusions}

In this paper we have presented a practical approach to automatic the repair of inconsistent ontologies. The key features of our approach are the following: (i) the semantics of the repair are simple, intuitive, formally grounded, and defined for all DLs; (ii) such semantics allow for tractable automatic ABox cleaning and consistent query rewriting in the case of OWL 2 QL ontologies; (iii) our experiments show that the approach is really scalable, and that very large ABoxes can be effectively repaired.

The work presented in this paper can be extended in several directions. First, the present implementation can be certainly further optimized. For instance, besided working with an annotated ABox representation, other optimizations are possible: one possibility which seems worth exploring is employing summarization techniques for ABox representation, as in [3]. Also, the consistent query rewriting technique can be certainly optimized to the aim of reducing the size of the reformulated query. Then, it would be very interesting to see whether the techniques presented in this paper can be extended to other tractable OWL profiles.

Acknowledgments. This research has been partially supported by the ICT Collaborative Project ACSI (Artifact-Centric Service Interoperation), funded by the EU under FP7 ICT Call 5, 2009.1.2, grant agreement n. FP7-257593.

\section{References}

1. Artale, A., Calvanese, D., Kontchakov, R., Zakharyaschev, M.: The DL-Lite family and relations. J. of Artificial Intelligence Research 36, 1-69 (2009)

2. Calvanese, D., De Giacomo, G., Lembo, D., Lenzerini, M., Rosati, R.: Tractable reasoning and efficient query answering in description logics: The DL-Lite family. J. of Automated Reasoning 39(3), 385-429 (2007)

3. Dolby, J., Fan, J., Fokoue, A., Kalyanpur, A., Kershenbaum, A., Ma, L., Murdock, J.W., Srinivas, K., Welty, C.A.: Scalable Cleanup of Information Extraction Data Using Ontologies. In: Aberer, K., Choi, K.-S., Noy, N., Allemang, D., Lee, K.-I., Nixon, L.J.B., Golbeck, J., Mika, P., Maynard, D., Mizoguchi, R., Schreiber, G., Cudré-Mauroux, P. (eds.) ISWC/ASWC 2007. LNCS, vol. 4825, pp. 100-113. Springer, Heidelberg (2007)

4. Hogan, A., Harth, A., Passant, A., Decker, S., Polleres, A.: Weaving the pedantic web. In: Proc. of 3rd Int. Workshop on Linked Data on the Web, LDOW 2010 (2010)

5. Lembo, D., Lenzerini, M., Rosati, R., Ruzzi, M., Savo, D.F.: Inconsistency-Tolerant Semantics for Description Logics. In: Hitzler, P., Lukasiewicz, T. (eds.) RR 2010. LNCS, vol. 6333, pp. 103-117. Springer, Heidelberg (2010) 
6. Lembo, D., Lenzerini, M., Rosati, R., Ruzzi, M., Savo, D.F.: Query Rewriting for Inconsistent DL-Lite Ontologies. In: Rudolph, S., Gutierrez, C. (eds.) RR 2011. LNCS, vol. 6902, pp. 155-169. Springer, Heidelberg (2011)

7. Poggi, A., Lembo, D., Calvanese, D., De Giacomo, G., Lenzerini, M., Rosati, R.: Linking Data to Ontologies. In: Spaccapietra, S. (ed.) Journal on Data Semantics X. LNCS, vol. 4900, pp. 133-173. Springer, Heidelberg (2008)

8. Wang, Z., Wang, K., Topor, R.W.: A new approach to knowledge base revision in DL-Lite. In: Proc. of AAAI 2010, AAAI Press (2010) 